\title{
Fast Algorithm for Rate-Based Optimal Error Protection of Embedded Codes
}

\author{
Vladimir Stanković, Raouf Hamzaoui, and Dietmar Saupe
}

\begin{abstract}
Embedded image codes are very sensitive to channel noise because a single bit error can lead to an irreversible loss of synchronization between the encoder and the decoder. Sherwood and Zeger introduced a powerful system that protects an embedded wavelet image code with a concatenation of a cyclic redundancy check coder for error detection and a rate-compatible punctured convolutional coder for error correction. For such systems, Chande and Farvardin proposed an unequal error protection strategy that maximizes the expected number of correctly received source bits subject to a target transmission rate. Noting that an optimal strategy protects successive source blocks with the same channel code, we give an algorithm that accelerates the computation of the optimal strategy of Chande and Farvardin by finding an explicit formula for the number of occurences of the same channel code. Experimental results with two competitive channel coders and a binary symmetric channel showed that the speed-up factor over the approach of Chande and Farvardin ranged from 2.82 to $\mathbf{4 4 . 7 6}$ for transmission rates between 0.25 and 2 bits per pixel.
\end{abstract}

Index Terms-Forward error correction (FEC), image coding, image communication, joint source-channel coding.

\section{INTRODUCTION}

W E CONSIDER a joint source-channel coding system for progressive image transmission that uses an embedded source coder and a finite set of channel codes with error detection and error protection capability [1], [2]. For example, the source coder may be the set partitioning in hierarchical trees (SPIHT) coder [3], JPEG2000 [4], or three-dimensional (3-D) SPIHT [5], and the channel coder may consist of the concatenation of an outer cyclic redundancy check (CRC) coder for error detection and an inner rate-compatible punctured convolutional (RCPC) coder for error correction [1]. The channel encoder transforms successive blocks of the source bitstream into a sequence of channel codewords, which are sent over a memoryless noisy channel. When the first decoding error is detected, the decoding is stopped, and the image is reconstructed from the

Paper approved by K. Illgner, the Editor for Speech, Image, Video, and Signal Processing of the IEEE Communications Society. Manuscript received August 8,2002 ; revised March 12, 2003. This paper was presented in part at MMSP'01, the IEEE Workshop on Multimedia Signal Processing, Cannes, France, October 2001.

V. Stanković is with the Department of Electrical Engineering, Texas A\&M University, College Station, TX 77843 USA (e-mail: stankovi@ee.tamu.edu).

R. Hamzaoui and D. Saupe are with the Department of Computer and Information Science, University of Konstanz, 78457 Konstanz, Germany (e-mail: hamzaoui@inf.uni-konstanz.de; saupe@inf.uni-konstanz.de).

Digital Object Identifier 10.1109/TCOMM.2003.819235

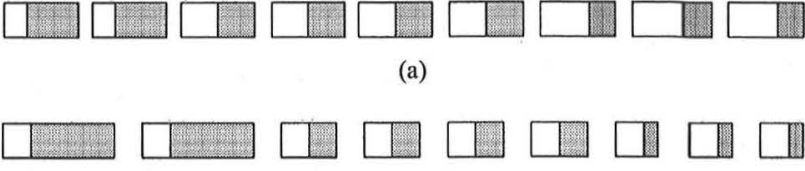

(b)

Fig. 1. (a) System used in this paper: Fixed-length channel codewords with variable-length information blocks. (b) System used in [1] and [2]: Fixed-length information blocks with variable-length channel codewords. For both systems, the white areas correspond to information bits and the shaded areas to protection bits. The channel code rate is $1 / 4$ for the first two codewords, $1 / 2$ for the next four codewords, and $2 / 3$ for the last three codewords.

correctly decoded codewords received up to that point. This is a reasonable approach for many embedded codes, including the SPIHT and JPEG2000 source codes, where, generally, a single bit error leads to a loss of synchronization between the encoder and the decoder [6]. In [1] and [2], the size of the blocks of information bits is fixed, while the channel codewords have a variable size. But, for many applications, including transmission in asynchronous transfer mode (ATM) networks, it is more suitable to fix the size of the channel codewords and let the information blocks have a variable size (see Fig. 1).

A challenging problem is to determine an optimal error protection for this system; that is, an assignment of the available channel codes (each specified by a different code rate) to the successive source blocks that minimizes the expected distortion subject to a target transmission rate. An alternative is to maximize the expected number of correctly received source bits for this target transmission rate. Though the solution based on maximizing the expected source rate is suboptimal in the distortion sense, the loss in reconstruction quality is small for efficient embedded coders (for a comparison, see [2] and [7] for the original system of [1] and [8] for the system considered in this paper). Moreover, the rate-based optimization has three advantages. First, an optimal protection can be found in linear time by adapting the algorithm proposed by Chande and Farvardin in the fixed-length information block framework ([2, Alg. 2]) to the fixed-length channel codeword one. Second, this optimal protection is independent of both the source coder and the test image. Thus, it can also be implemented at the receiver side, avoiding the need for overhead information. Third, if an optimal protection for a target transmission rate is known, then an optimal solution for all lower transmission rates can easily be derived without repeating the optimization. 
The strategy of Chande and Farvardin [2] determines successively the optimal code rate for each source block, starting from the last one. We propose an algorithm that reduces the time complexity of this strategy. The basic idea of our method is to see that an optimal solution shows long runs of the same code rate, which is typically due to the fact that the number of available code rates is much lower than the number of transmitted codewords, and to the property that in an optimal solution, the code rates are nondecreasing. Thus, one can save computation time by finding an explicit formula for the length of the code rate runs. For example, in Fig. 1 the solution has three runs of respective length two, four, and three.

The paper is organized as follows. In Section II, we introduce our terminology, adapt the algorithm of Chande and Farvardin ([2, Alg. 2]) to the fixed-length channel codeword context, and provide some properties of a rate-based optimal protection. In Section III, we give our algorithm and prove its optimality. In Section IV, we provide experimental results that illustrate the speed-up obtained with our approach for a binary symmetric channel and two different channel coders.

\section{Rate-Based Optimal Error Protection}

We consider a joint source-channel coding system where the source coder generates an embedded bitstream and the channel coder is given by a family $\mathcal{C}=\left\{C_{1}, \ldots, C_{m}\right\}$ of channel codes with error detection and error correction capability. These codes are such that all channel codewords have the same size $L$ (in bits). The set of corresponding code rates is denoted by $\mathcal{R}=$ $\left\{r_{1}, \ldots, r_{m}\right\}$, where $r_{1}<\cdots<r_{m}$. We assume without loss of generality that $p\left(r_{1}\right)<\cdots<p\left(r_{m}\right)<1$, where $p\left(r_{j}\right), j=1, \ldots, m$, is the probability of a decoding error when channel code $C_{j}$ is used. The system transforms successive blocks $b_{1}, b_{2}, \ldots$ of the embedded source bitstream into a sequence of channel codewords $c\left(b_{1}\right), c\left(b_{2}\right), \ldots$. The number of information bits in a block protected by code rate $r_{j}$ is thus $v\left(r_{j}\right)=\left\lfloor L r_{j}\right\rfloor$. Each channel packet consists of a single channel codeword (for simplicity, we ignore the header of the channel packet). For a given transmission bit budget $B$, the number of packets sent is $N=\lfloor B / L\rfloor$. An $N$-packet error-protection scheme (EPS) $R=\left(r_{k_{1}}, \ldots, r_{k_{N}}\right) \in \mathcal{R}^{N}$ protects the $i$ th source block $b_{i}, i=1, \ldots, N$, with a channel code rate $r_{k_{i}} \in \mathcal{R}$. For example, Fig. 1 shows a nine-packet EPS (1/4, $1 / 4,1 / 2,1 / 2,1 / 2,1 / 2,2 / 3,2 / 3,2 / 3)$.

The packets $c\left(b_{1}\right), c\left(b_{2}\right), \ldots, c\left(b_{N}\right)$ are sent successively over a memoryless noisy channel. Thus, we do not consider multiplexing and interleaving. As soon as a decoding error is detected, the decoding is stopped, and the image is reconstructed from the packets correctly decoded up to that point. Here we suppose that the probability of an undetected error is equal to zero. For $i=1, \ldots, N-1$, the number

$$
P_{i}(R)=p\left(r_{k_{i+1}}\right) \prod_{j=1}^{i}\left(1-p\left(r_{k_{j}}\right)\right)
$$

is the probability that no decoding errors occur in the first $i$ packets, with an error in the next one, $P_{0}(R)=p\left(r_{k_{1}}\right)$ is the probability that a decoding error occurs in the first packet, and $P_{N}(R)=\prod_{j=1}^{N}\left(1-p\left(r_{k_{j}}\right)\right)$ is the probability that no de- coding errors occur in the $N$ packets. For an $N$-packet EPS $R=\left(r_{k_{1}}, \ldots, r_{k_{N}}\right) \in \mathcal{R}^{N}$ the expected distortion is

$$
E_{N}[d](R)=\sum_{i=0}^{N} P_{i}(R) d_{i}(R)
$$

where $d_{0}$ is a constant, and for $i \geq 1, d_{i}(R)$ is the distortion from the reconstruction using the first $i$ information blocks.

Because minimizing $E_{N}[d]$ over $\mathcal{R}^{N}$ is time consuming, suboptimal solutions have been proposed [8], [9]. For example, one can replace the original problem by that of maximizing the expected number of correctly decoded source bits, which is

$$
E_{N}(R)=\sum_{i=1}^{N} P_{i}(R) V_{i}(R)
$$

where $V_{i}(R)=\sum_{j=1}^{i} v\left(r_{k_{j}}\right)$ is the number of information bits in the first $i$ packets. For simplicity of notation, we will sometimes drop the subscript $N$ from $E_{N}$ when the number of packets is clear from the context. An $N$-packet EPS $T \in \mathcal{R}^{N}$ is rate-optimal if for any $R \in \mathcal{R}^{N}$, we have $E_{N}(T) \geq E_{N}(R)$.

Chande and Farvardin [2] found a linear-time algorithm that computes a rate-optimal EPS for the original system of [1]. We now present this algorithm for the system considered in this paper [Fig. 1(a)]. Before presenting the algorithm, we provide several important results.

Lemma 1: Let $\left(r_{k_{1}}, r_{k_{2}}, \ldots, r_{k_{N}}\right) \in \mathcal{R}^{N}$ be an $N$-packet EPS. Then

$$
\begin{aligned}
E_{N}\left(r_{k_{1}}, r_{k_{2}}, \ldots, r_{k_{N}}\right)= & \left(1-p\left(r_{k_{1}}\right)\right)\left(v\left(r_{k_{1}}\right)\right. \\
& \left.+E_{N-1}\left(r_{k_{2}}, \ldots, r_{k_{N}}\right)\right) \\
= & E_{1}\left(r_{k_{1}}\right)+\left(1-p\left(r_{k_{1}}\right)\right) \\
& \times E_{N-1}\left(r_{k_{2}}, \ldots, r_{k_{N}}\right) .
\end{aligned}
$$

Proof: See Appendix I.

The above lemma gives a recurrence relation between $E_{N}\left(r_{k_{1}}, r_{k_{2}}, \ldots, r_{k_{N}}\right)$ and $E_{N-1}\left(r_{k_{2}}, \ldots, r_{k_{N}}\right)$.

Lemma 2: If the $(N-1)$-packet $\operatorname{EPS}\left(r_{2}^{*}, \ldots, r_{N}^{*}\right)$ is rate optimal, and if

$$
E_{N}\left(r_{1}^{*}, r_{2}^{*}, \ldots, r_{N}^{*}\right) \geq E_{N}\left(r_{k}, r_{2}^{*}, \ldots, r_{N}^{*}\right)
$$

for all $r_{k} \in \mathcal{R}$, then the $N$-packet EPS $\left(r_{1}^{*}, \ldots, r_{N}^{*}\right)$ is rate optimal.

Proof: See Appendix II.

This lemma shows how to derive a rate-optimal $N$-packet EPS from a rate-optimal $(N-1)$-packet EPS.

Proposition 1: If the $N$-packet EPS $\left(r_{1}^{*}, \ldots, r_{N}^{*}\right)$ is rate optimal, then the following holds.

1) For $1 \leq i \leq N-1$, the $(N-i)$-packet $\operatorname{EPS}\left(r_{i+1}^{*}, \ldots, r_{N}^{*}\right)$ is rate optimal.

2) $E_{i}\left(r_{N-i+1}^{*}, \ldots, r_{N-1}^{*}, r_{N}^{*}\right)$ is an increasing function of $i, i=1, \ldots, N$.

3) $r_{1}^{*} \leq \cdots \leq r_{N}^{*}$.

4) Let $1<k \leq N$. If the $(N-k+1)$-packet EPS $\left(r_{j_{k}}, r_{j_{k+1}}, \ldots, r_{j_{N}}\right)$ is rate optimal, then $r_{k-1}^{*} \leq r_{j_{k}}$. Proof: See Appendix III.

The most important result is part 3) of the proposition, which states that in a rate-optimal EPS, the code rates are 
nondecreasing with the packet number. Part 1) states that once a rate-optimal $N$-packet EPS is known, a rate-optimal $k$-packet $(k<N)$ EPS can easily be derived by truncating the first $(N-k)$ code rates. Part 4), which extends part 3$)$, is useful when a rate-optimal solution is not unique. The following example illustrates the importance of this result. Suppose that $\mathcal{R}=\left\{r_{1}, r_{2}, r_{3}, r_{4}\right\}$. Suppose that both $\left(r_{2}, r_{4}, r_{4}\right)$ and $\left(r_{3}, r_{4}, r_{4}\right)$ are rate optimal. Let $\left(r_{1}^{*}, \ldots, r_{4}^{*}\right)$ be any rate-optimal four-packet EPS. Then $r_{1}^{*} \leq \min \left(r_{2}, r_{3}\right)=r_{2}$.

Note that 2) is not necessarily satisfied if the EPS is not rate optimal.

Lemma 1, Lemma 2, and Proposition 1.3 can be found in [2]. However, no proofs were provided, and all results were given for the particular system considered there.

To find a rate-optimal $N$-packet EPS, the approach of [2] consists of determining first a rate-optimal one-packet EPS, and to complete the solution by repetitive use ( $N-1$ times) of Lemma 2 together with Proposition 1.3. This gives the following algorithm.

Proposition 2: Let $N$ be a positive integer and let $\mathcal{R}$ be the set of $m$ code rates $r_{1}<\cdots<r_{m}$. A rate-optimal $N$-packet EPS $\left(r_{1}^{*}, \ldots, r_{N}^{*}\right)$ can be computed as follows.

1) Set $i=1$ and $j_{1}=\arg \max _{k=1, \ldots, m} E_{1}\left(r_{k}\right)$.

2) If $i=N$, then set $\left(r_{1}^{*}, \ldots, r_{N}^{*}\right)=\left(r_{j_{i}}, \ldots, r_{j_{1}}\right)$ and stop. If $r_{j_{i}}=r_{1}$, then set $\left(r_{1}^{*}, \ldots, r_{N}^{*}\right)=\left(r_{j_{i}}, \ldots, r_{j_{i}}, r_{j_{i-1}}, \ldots, r_{j_{1}}\right)$ and stop.

3) Set $i=i+1, j_{i}=$ $\arg \max _{k=1, \ldots, m} r_{k} \leq r_{j_{i-1}} E_{i}\left(r_{k}, r_{j_{i-1}}, \ldots, r_{j_{1}}\right)$, and go to step 2 .

\section{FASt Algorithm fOR RATE-Optimal Protection}

In this section, we show how to accelerate the algorithm of Proposition 2. We first introduce some notations and technical lemmas that are needed to prove the optimality of our algorithm.

For $r_{j}, r_{k} \in \mathcal{R}$ and integer $t \geq 1$, let $q\left(r_{j}\right)=1-p\left(r_{j}\right)$

$$
T\left(r_{j}, t\right)=\frac{E_{1}\left(r_{j}\right)\left(1-q\left(r_{j}\right)^{t}\right)}{1-q\left(r_{j}\right)}
$$

and

$$
M\left(r_{k}, r_{j}\right)=\frac{E_{1}\left(r_{k}\right)\left(1-q\left(r_{j}\right)\right)-E_{1}\left(r_{j}\right)\left(1-q\left(r_{k}\right)\right)}{\left(q\left(r_{k}\right)-q\left(r_{j}\right)\right)\left(1-q\left(r_{j}\right)\right)} .
$$

Then we have:

Lemma 3: Let $N \geq 1$. Then for any positive integers $t_{0}, \ldots, t_{i}, N=t_{0}+\cdots+t_{i}$, and channel code rates $r_{j_{0}}, \ldots, r_{j_{i}} \in \mathcal{R}, p\left(r_{j_{k}}\right) \neq 0,0 \leq k \leq i$

$$
\begin{aligned}
E_{N} & (\underbrace{r_{j_{i}}, \ldots, r_{j_{i}}}_{t_{i}}, \ldots, \underbrace{r_{j_{0}}, \ldots, r_{j_{0}}}_{t_{0}}) \\
& =\sum_{l=0}^{i} E_{t_{l}}\left(r_{j_{l}}, \ldots, r_{j_{l}}\right) \times \prod_{k=l+1}^{i} q\left(r_{j_{k}}\right)^{t_{k}} \\
& =\sum_{l=0}^{i} T\left(r_{j_{l}}, t_{l}\right) \times \prod_{k=l+1}^{i} q\left(r_{j_{k}}\right)^{t_{k}} .
\end{aligned}
$$

Proof: See Appendix IV.

Let $r_{j_{0}}, \ldots, r_{j_{i}} \in \mathcal{R}$ with $p\left(r_{j_{i}}\right) \neq 0$ and let $t_{0}, \ldots, t_{i-1}$ be positive integers. Let $A_{i}=E_{1}\left(r_{j_{i}}\right) /\left(1-q\left(r_{j_{i}}\right)\right), B_{0}=0$, and

$$
B_{i}=E_{t_{0}+\cdots+t_{i-1}}(\underbrace{r_{j_{i-1}}, \ldots, r_{j_{i-1}}}_{t_{i-1}}, \ldots, \underbrace{r_{j_{0}}, \ldots, r_{j_{0}}}_{t_{0}})
$$

for $i \geq 1$. For $k \in\{1, \ldots, m\}, t \in \mathbb{R}$, and $i \geq 0$, let

$$
\psi_{i, k}(t)=\left(A_{i}-B_{i}\right) q\left(r_{j_{i}}\right)^{t-1}-M\left(r_{k}, r_{j_{i}}\right) .
$$

Lemma 4: For $i \geq 0$, let $r_{j_{0}}, \ldots, r_{j_{i}} \in \mathcal{R}$ with $p\left(r_{j_{i}}\right) \neq$ 0 , and let $t_{0}, \ldots, t_{i-1}$ be positive integers. Suppose that $k \in$ $\{1, \ldots, m\}$ and $r_{k}<r_{j_{i}}$. Then $\psi_{i, k}(t)$ and

$$
\begin{aligned}
E(r_{j_{i}}, \underbrace{r_{j_{i}}, \ldots, r_{j_{i}}}_{t-1}, \underbrace{r_{j_{i-1}}, \ldots, r_{j_{i-1}}}_{t_{i-1}}, \ldots, \underbrace{r_{j_{0}}, \ldots, r_{j_{0}}}_{t_{0}}) \\
-E(r_{k}, \underbrace{r_{j_{i}}, \ldots, r_{j_{i}}}_{t-1}, \underbrace{r_{j_{i-1}}, \ldots, r_{j_{i-1}}}_{t_{i-1}}, \ldots, \underbrace{r_{j_{0}}, \ldots, r_{j_{0}}}_{t_{0}})
\end{aligned}
$$

have the same sign for any positive integer $t$.

Proof: See Appendix V.

Lemma 5: For $i \geq 1$, let $r_{j_{0}}, \ldots, r_{j_{i}} \in \mathcal{R}$ with $p\left(r_{j_{i}}\right) \neq 0$, and let $t_{0}, \ldots, t_{i-1}$ be positive integers. If $(\underbrace{r_{j_{i-1}}, \ldots, r_{j_{i-1}}}_{t_{i-1}}, \ldots, \underbrace{r_{j_{0}}, \ldots, r_{j_{0}}}_{t_{0}})$ is a rate-optimal EPS, and if $\psi_{i-1, j_{i}}\left(t_{i-1}+1\right)<0$, then $A_{i}-B_{i}>0$.

Proof: See Appendix VI.

We now introduce our algorithm. The basic idea is to compute the run length of a code rate ahead of time.

Proposition 3: Let $N$ be a positive integer. Then a rate-optimal $N$-packet EPS is $(\underbrace{r_{j_{n}}, \ldots, r_{j_{n}}}_{t}, \ldots, \underbrace{r_{j_{0}}, \ldots, r_{j_{0}}}_{t_{0}})$, where $n \geq 0, r_{j_{0}}, \ldots, r_{j_{n}} \in \mathcal{R}$, and $t_{0}, \ldots, t_{n} \geq 1$ are given by the following algorithm.

1) Set $j_{0}=\arg \max _{k=1, \ldots, m} E_{1}\left(r_{k}\right)$ (if the argument is not unique, choose the one which corresponds to the smallest code rate). Set $i=0$.

2) If $r_{j_{i}}=r_{1}$, set $n=i, t_{n}=N-\sum_{p=0}^{i-1} t_{p}$ and stop.

3) For all $k \in\{1, \ldots, m\}, r_{k}<r_{j_{i}}$, if $a_{i, k}=\log \left(M\left(r_{k}, r_{j_{i}}\right) /\left(A_{i}-B_{i}\right)\right) / \log q\left(r_{j_{i}}\right)+1$ exists and is finite, then set $t_{i, k}=\left\lfloor a_{i, k}\right\rfloor$. Otherwise, set $t_{i, k}=N-\sum_{p=0}^{i-1} t_{p}$. Let $t_{i}=\min _{k, r_{k}<r_{j_{i}}} t_{i, k}$.

4) If $N \leq \sum_{p=0}^{i} t_{p}$, set $n=i, r_{j_{n}}=r_{j_{i}}, t_{n}=$ $N-\sum_{p=0}^{i-1} t_{p}$ and stop. Otherwise, set

$j_{i+1}=\arg \max _{k, r_{k}<r_{j_{i}}, t_{i, k}=t_{i}} E(r_{k}, \underbrace{r_{j_{i}}, \ldots, r_{j_{i}}}_{t_{i}}, \ldots, \underbrace{r_{j_{0}}, \ldots, r_{j_{0}}}_{t_{0}})$

(if the argument is not unique, choose the one which corresponds to the 
smallest code rate), set $i=i+1$ and go to Step 2 .

Proof: If $r_{j_{0}}=r_{1}$, then the algorithm yields the EPS $(\underbrace{r_{j_{0}}, \ldots, r_{j_{0}}})$, which is rate optimal by Lemma 2 and Propo$N$

sition 1.3. Suppose now that $r_{j_{0}} \neq r_{1}$. Let $k \in\{1, \ldots, m\}$ with $r_{k}<r_{j_{0}}$. Because $A_{0}-B_{0}=A_{0}>0$, the function $\psi_{0, k}$ is decreasing on $\mathbb{R}$ from $+\infty$ to $-M\left(r_{k}, r_{j_{0}}\right)$. If $a_{0, k} \notin \mathbb{R}$, then $t_{0, k}=N-\sum_{p=0}^{i-1} t_{p}=N \geq 1$. If $a_{0, k} \in \mathbb{R}$, then $\psi_{0, k}(1) \geq 0$ by Lemma 4. Moreover, $\psi_{0, k}\left(a_{0, k}\right)=0$. Thus, $a_{0, k} \geq 1$, which gives $t_{0, k} \geq 1$. Hence, $t_{0} \geq 1$. Now let $t$ be an integer such that $1 \leq t \leq t_{0}$. If $a_{0, k} \in \mathbb{R}$, then $\psi_{0, k}(t) \geq$ $\psi_{0, k}\left(t_{0}\right) \geq \psi_{0, k}\left(t_{0, k}\right) \geq \psi_{0, k}\left(a_{0, k}\right)=0$. If $a_{0, k} \notin \mathbb{R}$, then $-M\left(r_{k}, r_{j_{0}}\right) \geq 0$, and here also $\psi_{0, k}(t) \geq 0$. Thus, Lemma 4 gives

$$
E(r_{j_{0}}, \underbrace{r_{j_{0}}, \ldots, r_{j_{0}}}_{t-1}) \geq E(r_{k}, \underbrace{r_{j_{0}}, \ldots, r_{j_{0}}}_{t-1})
$$

for all $k \in\{1, \ldots, m\}$ with $r_{k}<r_{j_{0}}$. If $N \leq \sum_{p=0}^{i} t_{p}=t_{0}$, then the algorithm yields the EPS $(\underbrace{r_{j_{0}}, \ldots, r_{j_{0}}}_{N})$, which is rate optimal by inequality (4), Lemma 2, and Proposition 1.3. We assume now that $N>\sum_{p=0}^{i} t_{p}=t_{0}$. We have $t_{0, j_{1}}=t_{0}$. Since $t_{0, j_{1}}+1>a_{0, j_{1}}$, we get $\psi_{0, j_{1}}\left(t_{0}+1\right)<\psi_{0, j_{1}}\left(a_{0, j_{1}}\right)=0$, which gives

$$
E(r_{j_{1}}, \underbrace{r_{j_{0}}, \ldots, r_{j_{0}}}_{t_{0}})>E(\underbrace{r_{j_{0}}, \ldots, r_{j_{0}}}_{t_{0}+1})
$$

by Lemma 4 and $A_{1}-B_{1}>0$ by Lemma 5 . On the other hand, $t_{0}+1 \leq a_{0, k}$ for all $k$ such that $t_{0, k} \neq t_{0}$. Thus, $\psi_{0, k}\left(t_{0}+1\right) \geq$ $\psi_{0, k}\left(a_{0, k}\right)=0$, or

$$
E(\underbrace{r_{j_{0}}, \ldots, r_{j_{0}}}_{t_{0}+1}) \geq E(r_{k}, \underbrace{r_{j_{0}}, \ldots, r_{j_{0}}}_{t_{0}})
$$

for all $k$ such that $t_{0, k} \neq t_{0}$. From inequalities (5) and (6), we obtain

$$
E(r_{j_{1}}, \underbrace{r_{j_{0}}, \ldots, r_{j_{0}}}_{t_{0}}) \geq E(r_{k}, \underbrace{r_{j_{0}}, \ldots, r_{j_{0}}}_{t_{0}})
$$

for all $k$ such that $t_{0, k} \neq t_{0}$. Moreover, by construction, inequality (7) holds for all $k$ such that $r_{k}<r_{j_{0}}$ and $t_{0, k}=t_{0}$. Thus, inequality (7) is true for all $k$ such that $r_{k}<r_{j_{0}}$. Using the same approach as for (4), we can prove that for $i=1,2, \ldots, n$, if $1 \leq t \leq t_{i}$, then for all $r_{k} \leq r_{j_{i}}$

$$
\begin{aligned}
& E_{t_{0}+\cdots+t_{i-1}+t}(r_{j_{i}}, \underbrace{r_{j_{i}}, \ldots, r_{j_{i}}}_{t-1}, \ldots, \underbrace{r_{j_{0}}, \ldots, r_{j_{0}}}_{t_{0}}) \\
& \geq E_{t_{0}+\cdots+t_{i-1}+t}(r_{k}, \underbrace{r_{j_{i}}, \ldots, r_{j_{i}}}_{t-1}, \ldots, \underbrace{r_{j_{0}}, \ldots, r_{j_{0}}}_{t_{0}})
\end{aligned}
$$

and with the same approach as for (7), we can show that for $i=2, \ldots, n$

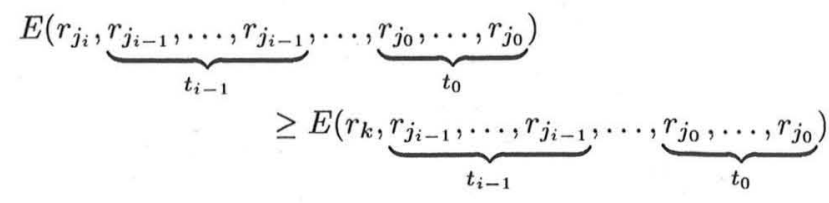

for $r_{k} \leq r_{j_{i-1}}$. Thus, Lemma 2 and Proposition 1.3 show that the EPS yielded by the algorithm is optimal. When in Step 1 or 4 the argument is not unique, we choose the smallest code rate because this choice reduces the complexity of the algorithm by excluding more code rates from the next optimization steps. Note that Proposition 1.4 justifies why the choice of the smallest code rate does not violate the optimality of the algorithm.

In Step 3, $B_{i}$ should be computed using Lemma 3. In Step 4, $E(r_{k}, \underbrace{r_{j_{i}}, \ldots, r_{j_{i}}}_{t_{i}}, \ldots, \underbrace{r_{j_{0}}, \ldots, r_{j_{0}}}_{t_{0}})$ can easily be computed from $B_{i}$ by Lemma 1. Finally, if in Step 3 there exists a unique $k$ such that $\left\lfloor a_{i, k}\right\rfloor=t_{i}$, then $j_{i+1}=k$.

In Fig. 1, for example, the run lengths of the code rates are $t_{0}=3, t_{1}=4$, and $t_{2}=2$ with $r_{j_{0}}=2 / 3, r_{j_{1}}=1 / 2$, and $r_{j_{2}}=1 / 4$.

\section{RESULTS}

In this section, we compare the time complexity of our algorithm to that of Proposition 2 for a binary symmetric channel and two channel coders. We recall that $N$ and $L$ denote the number of packets sent and the length of the channel codeword, respectively. Thus, the transmission rate is $R_{T}=N L / n^{2}$ bits per pixel (bpp) for $n \times n$ images.

In the first experiment, the channel coder was a concatenation of a CRC-32 coder and a rate-compatible punctured turbo (RCPT) coder [10]. The generator polynomial of the CRC code was $(32,26,23,22,16,12,11,10,8,7,5,4,2$, $1,0)$. The turbo coder consisted of two identical recursive systematic convolutional coders [11] with memory length four and generator polynomials $(31,27)$ octal. The code rate of the mother code was $20 / 60=1 / 3$, and the puncturing rate was 20 , yielding 41 possible channel code rates. The length of a channel codeword was equal to $L=2048$ bits, consisting of a variable number of source bits, $32 \mathrm{CRC}$ bits, 4 bits to set the turbo encoder into a state of all zeros, and protection bits. We used iterative maximum a posteriori decoding, which was stopped if no correct sequence was found after 20 iterations. When the source coder is the SPIHT coder, this system yields state-of-the-art rate-distortion performance (see [12, Table 2]). For each bit-error rate (BER) and each code rate, the probability of a packet decoding error was computed with 50000 Monte-Carlo simulations. Channel code rates for which this probability was equal to one were removed. The set of used code rates was $\mathcal{R}=\{20 / 40,20 / 38,20 / 36,20 / 35\}$ for $\mathrm{BER}=0.05$ and $\mathcal{R}=\{20 / 56,20 / 54,20 / 52,20 / 51$, $20 / 50,20 / 48,20 / 47,20 / 46\}$ for BER $=0.1$. Using the notation of Proposition 3, the rate-optimal solution for BER $=0.05$ is given by $r_{j_{0}}=20 / 35, t_{0}=56, r_{j_{1}}=20 / 36, t_{1}=225$, 
and $r_{j_{2}}=20 / 40, t_{2}=N-\left(t_{0}+t_{1}\right)$. For example, suppose that we want to send a $512 \times 512$ image at a transmission rate of $1 \mathrm{bpp}$. Then $N=128$ packets have to be sent. Whereas the approach of Chande and Farvardin determines the optimal code rate for each of the 128 corresponding source blocks, our strategy needs only two steps. The first one identifies the run of 56 source blocks (starting from the end) that should be protected by code rate $20 / 35$, and the second step finds the run of 72 source blocks that should be protected by code rate 20/36.

For BER $=0.1$, the rate-optimal solution is given by $r_{j_{0}}=$ $20 / 48, t_{0}=11, r_{j_{1}}=20 / 50, t_{1}=30, r_{j_{2}}=20 / 52, t_{2}=365$, and $r_{j_{3}}=20 / 56, t_{3}=N-\left(t_{0}+t_{1}+t_{2}\right)$.

In the second experiment, the channel coder was a concatenation of a CRC-16 coder and an RCPC coder. The generator polynomial of the CRC code was as in [1]. The RCPC codes, taken from [13], had a mother code with memory length six, generator polynomial $(147,163,135,135)$ octal, and code rate $1 / 4$. The puncturing period was 8 , yielding 25 possible channel code rates. The channel codeword length was $L=512$ bits, consisting of information bits, 16 CRC detection bits, 6 bits for setting the convolutional encoder into a state of all zeros, and protection bits. The decoding was based on a tree-trellis search technique with a list Viterbi algorithm [14]. If the path selected by the Viterbi decoder was not declared correct by the CRC test, the decoder selected the next best path. This procedure was repeated until the CRC test was passed or 100 paths were considered. For each BER and each code rate, the probability of a packet decoding error was computed with 100000 Monte-Carlo simulations. The set of used rates was $\mathcal{R}=\{8 / 20,8 / 18,8 / 16,8 / 14,8 / 12,8 / 10\}$ for BER $=0.05$ and $\mathcal{R}=\{8 / 28,8 / 27,8 / 26,8 / 25,8 / 24,8 / 22,8 / 20,8 / 18\}$ for $\mathrm{BER}=0.1$. The rate-optimal solution for $\mathrm{BER}=0.05$ is given by $r_{j_{0}}=8 / 14, t_{0}=4, r_{j_{1}}=8 / 16, t_{1}=86, r_{j_{2}}=8 / 18$, $t_{2}=5863$, and $r_{j_{3}}=8 / 20, t_{3}=N-\left(t_{0}+t_{1}+t_{2}\right)$. For $\mathrm{BER}=0.1$, the rate-optimal solution is given by $r_{j_{0}}=8 / 20$, $t_{0}=3, r_{j_{1}}=8 / 22, t_{1}=19, r_{j_{2}}=8 / 24, t_{2}=113$, $r_{j_{3}}=8 / 25, t_{3}=333, r_{j_{4}}=8 / 27, t_{4}=494$, and $r_{j_{5}}=8 / 28$, $t_{5}=N-\left(t_{0}+t_{1}+t_{2}+t_{3}+t_{4}\right)$.

Fig. 2 compares the time complexity of our algorithm to that of Proposition 2 for various target transmission rates. The transmission rate is given for $512 \times 512$ images. The CPU time was measured on a $195 \mathrm{MHz}$ MIPS R10000 processor of an SGI Origin200. The algorithm of Proposition 2 is already fast. For example, for RCPC codes at transmission rate $2 \mathrm{bpp}$ and channel $\mathrm{BER}=0.1$, it found an optimal solution in $1.02 \mathrm{~ms}$. Except for very low transmission rates, our algorithm was always faster (i.e., the speed-up factor was greater than one). Generally, the speed-up factor increased with the transmission rate. However, due to the complex formula for $a_{i, k}$, which is needed to compute the length of a code rate run, a drop in the speed-up factor happened each time our algorithm needed to compute a new code rate run. This also explains why our algorithm was slower at very low transmission rates. Indeed, when only a few packets are present, the cost for computing a code rate run length outweighs the cost of computing the code rate for each channel codeword. Also when the smallest code rate was selected, both algorithms stopped and no further speed-up occurred [see the extremity of the graph corresponding to BER $=0.1$ in Fig. 2(b)]. Fi-

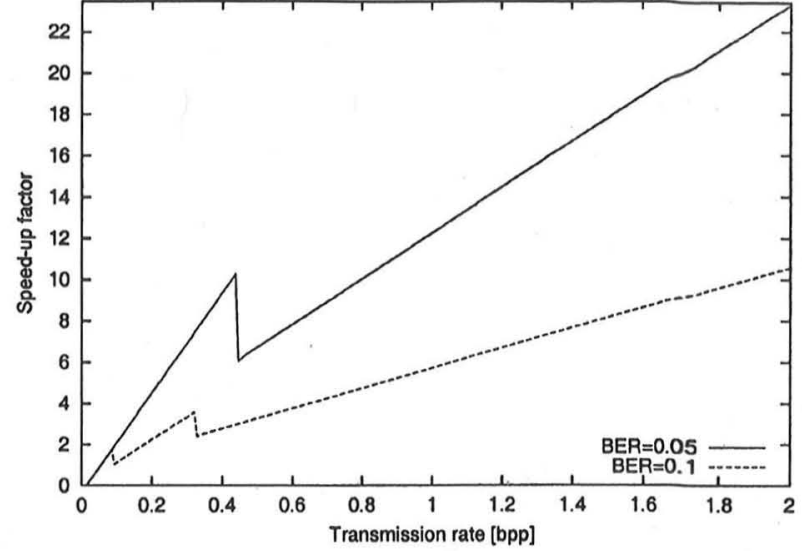

(a)

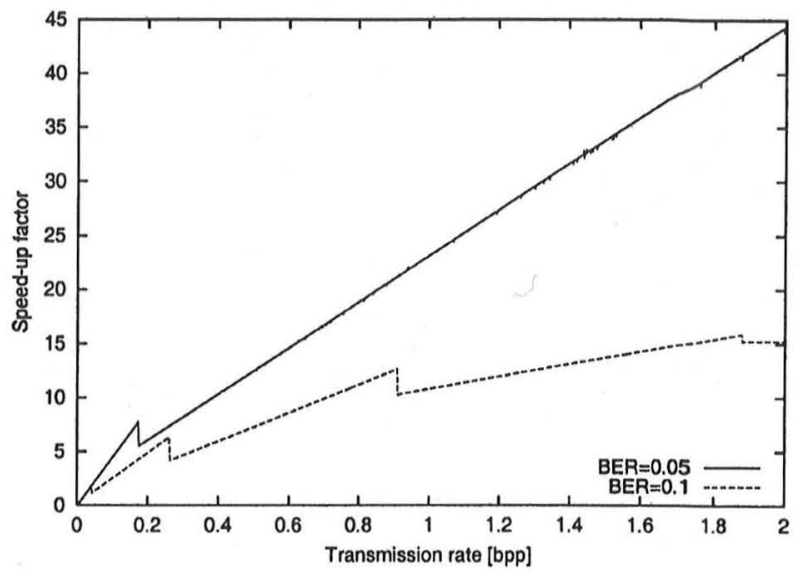

(b)

Fig. 2. Average speed-up factors of our algorithm over the algorithm of Proposition 2 for various target transmission rates. Results are given for two channel coders, (a) RCPT and (b) RCPC, and two BERs (0.05 and 0.1).

nally, the speed-up factor was higher for BER $=0.05$ than for $\mathrm{BER}=0.1$ because there were fewer code rate changes with the lower BER.

\section{CONCLUSION}

We considered joint source-channel coding for progressive image transmission in memoryless noisy channels. We showed how to speed up a strategy of Chande and Farvardin [2] that assigns channel codes to the source blocks, such that the expected number of correctly received source bits is maximized subject to a target transmission rate. Whereas the original algorithm must determine the optimal channel code for each source block, our method determines ahead of time the number of successive source blocks that should be protected with the same channel code. Though the original algorithm is already fast, accelerating it is especially desirable in two applications. The first one is when the channel conditions change during transmission and, consequently, a new optimal protection has to be computed online. The second one is when it is used as an initial step in the local search algorithm of [8], which can find a near-optimal solution to the problem of minimizing the expected distortion (1). 
APPENDIX I

\section{Proof of Lemma 1}

Let $R=\left(r_{k_{1}}, \ldots, r_{k_{N}}\right)$. Then

$$
\begin{aligned}
E_{N}(R) & =v\left(r_{k_{1}}\right) \sum_{i=1}^{N} P_{i}(R)+\sum_{i=2}^{N} P_{i}(R) \sum_{j=2}^{i} v\left(r_{k_{j}}\right) \\
& =v\left(r_{k_{1}}\right)\left(1-p\left(r_{k_{1}}\right)\right)+\sum_{i=2}^{N} P_{i}(R) \sum_{j=2}^{i} v\left(r_{k_{j}}\right)
\end{aligned}
$$

and the result follows from the equality

$$
\sum_{i=2}^{N} P_{i}(R) \sum_{j=2}^{i} v\left(r_{k_{j}}\right)=\left(1-p\left(r_{k_{1}}\right)\right) E_{N-1}\left(r_{k_{2}}, \ldots, r_{k_{N}}\right) .
$$

\section{APPENDIX II}

\section{Proof of Lemma 2}

Suppose that the $(N-1)$-packet EPS $\left(r_{2}^{*}, \ldots, r_{N}^{*}\right)$ is rate optimal. Let $r_{k_{1}}, r_{k_{2}}, \ldots, r_{k_{N}} \in \mathcal{R}$. Then

$$
\begin{aligned}
E_{N}\left(r_{1}^{*}, r_{2}^{*}, \ldots, r_{N}^{*}\right) \geq & E_{N}\left(r_{k_{1}}, r_{2}^{*}, \ldots, r_{N}^{*}\right) \\
= & \left(1-p\left(r_{k_{1}}\right)\right) \\
& \times\left(v\left(r_{k_{1}}\right)+E_{N-1}\left(r_{2}^{*}, \ldots, r_{N}^{*}\right)\right) \\
\geq & \left(1-p\left(r_{k_{1}}\right)\right) \\
& \times\left(v\left(r_{k_{1}}\right)+E_{N-1}\left(r_{k_{2}}, \ldots, r_{k_{N}}\right)\right) \\
= & E_{N}\left(r_{k_{1}}, r_{k_{2}}, \ldots, r_{k_{N}}\right)
\end{aligned}
$$

which shows that the $N$-packet EPS $\left(r_{1}^{*}, \ldots, r_{N}^{*}\right)$ is rate optimal.

\section{APPENDIX III}

\section{Proof of Proposition 1}

1) Let $r_{k_{2}}, \ldots, r_{k_{N}} \in \mathcal{R}$. Then $E_{N}\left(r_{1}^{*}, r_{2}^{*}, \ldots, r_{N}^{*}\right) \geq$ $E_{N}\left(r_{1}^{*}, r_{k_{2}}, \ldots, r_{k_{N}}\right)$. By Lemma 1 , this inequality can be expressed as

$$
\begin{aligned}
E_{1}\left(r_{1}^{*}\right)+\left(1-p\left(r_{1}^{*}\right)\right) E_{N-1}\left(r_{2}^{*}, \ldots, r_{N}^{*}\right) \\
\\
\geq E_{1}\left(r_{1}^{*}\right)+\left(1-p\left(r_{1}^{*}\right)\right) E_{N-1}\left(r_{k_{2}}, \ldots, r_{k_{N}}\right)
\end{aligned}
$$

or $E_{N-1}\left(r_{2}^{*}, \ldots, r_{N}^{*}\right) \geq E_{N-1}\left(r_{k_{2}}, \ldots, r_{k_{N}}\right)$. Similarly, $E_{N-i}\left(r_{i+1}^{*}, \ldots, r_{N}^{*}\right) \geq E_{N-i}\left(r_{k_{i+1}}, \ldots, r_{k_{N}}\right)$ for $i=2, \ldots, N-1$.

2) By 1$)$, the $(i+1)$-packet EPS $\left(r_{N-i}^{*}, \ldots, r_{N-1}^{*}, r_{N}^{*}\right)$ is rate optimal. Thus, for $r_{j} \in \mathcal{R}$

$$
\begin{aligned}
& E_{i+1}\left(r_{N-i}^{*}, \ldots, r_{N-1}^{*}, r_{N}^{*}\right) \\
& \quad \geq E_{i+1}\left(r_{N-i+1}^{*}, \ldots, r_{N-1}^{*}, r_{N}^{*}, r_{j}\right) \\
& \quad=E_{i}\left(r_{N-i+1}^{*}, \ldots, r_{N-1}^{*}, r_{N}^{*}\right)+E\left(r_{j}\right) \prod_{k=0}^{i-1}\left(1-p\left(r_{N-k}^{*}\right)\right) \\
& \quad>E_{i}\left(r_{N-i+1}^{*}, \ldots, r_{N-1}^{*}, r_{N}^{*}\right)
\end{aligned}
$$

where (8) is obtained by successive use of (3).
3) One can express $\left(r_{1}^{*}, \ldots, r_{N}^{*}\right)$ as $(\underbrace{r_{j_{n}}, \ldots, r_{j_{n}}}_{t_{n}}, \ldots, \underbrace{r_{j_{0}}, \ldots, r_{j_{0}}}_{t_{0}})$ with $n \geq 0$, $t_{i} \geq 1$ for $i=0, \ldots, n, N=\sum_{i=0}^{n} t_{i}$, and $r_{j_{i}} \neq r_{j_{i+1}}, 0 \leq i \leq n-1$. Due to 1), we have $E_{t_{0}+1}\left(r_{j_{1}}, r_{j_{0}}, \ldots, r_{j_{0}}\right) \geq E_{t_{0}+1}\left(r_{j_{0}}, r_{j_{0}}, \ldots, r_{j_{0}}\right)$, which can be written $E_{1}\left(r_{j_{0}}\right)-E_{1}\left(r_{j_{1}}\right) \leq$ $\left(p\left(r_{j_{0}}\right)-p\left(r_{j_{1}}\right)\right) E_{t_{0}}\left(r_{j_{0}}, \ldots, r_{j_{0}}\right)$. Thus, since $E_{1}\left(r_{j_{0}}\right) \geq E_{1}\left(r_{j_{1}}\right)$, we get $p\left(r_{j_{1}}\right)<p\left(r_{j_{0}}\right)$, and hence, $r_{j_{1}}<r_{j_{0}}$. Similarly, the inequality $E_{t_{0}+1}\left(r_{j_{1}}, r_{j_{0}}, \ldots, r_{j_{0}}\right) \geq E_{t_{0}+1}\left(r_{j_{2}}, r_{j_{0}}, \ldots, r_{j_{0}}\right)$ gives

$$
\begin{aligned}
& E_{1}\left(r_{j_{1}}\right)-E_{1}\left(r_{j_{2}}\right) \geq\left(p\left(r_{j_{1}}\right)-p\left(r_{j_{2}}\right)\right) E_{t_{0}}\left(r_{j_{0}}, \ldots, r_{j_{0}}\right) \\
& \text { and } E_{t_{0}+t_{1}+1}\left(r_{j_{1}}, r_{j_{1}}, \ldots, r_{j_{1}}, r_{j_{0}}, \ldots, r_{j_{0}}\right) \leq \\
& E_{t_{0}+t_{1}+1}\left(r_{j_{2}}, r_{j_{1}}, \ldots, r_{j_{1}}, r_{j_{0}}, \ldots, r_{j_{0}}\right) \text { gives }
\end{aligned}
$$

$$
\begin{aligned}
E_{1}\left(r_{j_{1}}\right)-E_{1}\left(r_{j_{2}}\right) \leq & \left(p\left(r_{j_{1}}\right)-p\left(r_{j_{2}}\right)\right) \\
& \times E_{t_{0}+t_{1}}\left(r_{j_{1}}, \ldots, r_{j_{1}}, r_{j_{0}}, \ldots, r_{j_{0}}\right) .
\end{aligned}
$$

From 2), we have $E_{t_{0}+t_{1}}\left(r_{j_{1}}, \ldots, r_{j_{1}}, r_{j_{0}}, \ldots, r_{j_{0}}\right)>$ $E_{t_{0}}\left(r_{j_{0}}, \ldots, r_{j_{0}}\right)$. Thus, inequalities (9) and (10) can be simultaneously fulfilled only when $r_{j_{2}}<r_{j_{1}}$. The same method can be used to show that $r_{j_{i+1}}<r_{j_{i}}$ for $2 \leq i \leq$ $N-1$.

4) We have

$$
\begin{aligned}
& E\left(r_{1}^{*}, \ldots, r_{k-1}^{*}, r_{j_{k}}, r_{j_{k+1}}, \ldots, r_{j_{N}}\right) \\
& =E\left(r_{1}^{*}, \ldots, r_{k-1}^{*}\right)+E\left(r_{j_{k}}, \ldots, r_{j_{N}}\right) \\
& \quad \times \prod_{i=1}^{k-1}\left(1-p\left(r_{i}^{*}\right)\right) \\
& \geq E\left(r_{1}^{*}, \ldots, r_{k-1}^{*}\right)+E\left(r_{k}^{*}, \ldots, r_{N}^{*}\right) \\
& \quad \times \prod_{i=1}^{k-1}\left(1-p\left(r_{i}^{*}\right)\right) \\
& =E\left(r_{1}^{*}, r_{2}^{*}, \ldots, r_{N}^{*}\right) .
\end{aligned}
$$

Thus, the $N$-packet EPS $\left(r_{1}^{*}, \ldots, r_{k-1}^{*}, r_{j_{k}}, \ldots, r_{j_{N}}\right)$ is rate optimal, which gives the desired inequality by 3 ).

\section{APPENDIX IV}

\section{Proof of Lemma 3}

Let $E=E_{N}(\underbrace{r_{j_{i}}, \ldots, r_{j_{i}}}_{t_{i}}, \ldots, \underbrace{r_{j_{0}}, \ldots, r_{j_{0}}}_{t_{0}})$. Then as in (8)

$$
\begin{aligned}
E= & E_{t_{i}}\left(r_{j_{i}}, \ldots, r_{j_{i}}\right)+\left[q\left(r_{j_{i}}\right)\right]^{t_{i}} \\
& \times E_{N-t_{i}}(\underbrace{r_{j_{i-1}}, \ldots, r_{j_{i-1}}}_{t_{i-1}}, \ldots, \underbrace{r_{j_{0}}, \ldots, r_{j_{0}}}_{t_{0}}) \\
= & E_{t_{i}}\left(r_{j_{i}}, \ldots, r_{j_{i}}\right)+\left[q\left(r_{j_{i}}\right)\right]^{t_{i}} E_{t_{i-1}}\left(r_{j_{i-1}}, \ldots, r_{j_{i-1}}\right) \\
& +\left[q\left(r_{j_{i}}\right)\right]^{t_{i}}\left[q\left(r_{j_{i-1}}\right)\right]^{t_{i-1}} \\
& \times E_{N-t_{i}-t_{i-1}}(\underbrace{r_{j_{i-2}}, \ldots, r_{j_{i-2}}}_{t_{i-2}}, \ldots, \underbrace{r_{j_{0}}, \ldots, r_{j_{0}}}_{t_{0}}) \\
= & \sum_{l=0}^{i} E_{t_{l}}\left(r_{j_{l}}, \ldots, r_{j_{l}}\right) \prod_{k=l+1}^{i} q\left(r_{j_{k}}\right)^{t_{k}} .
\end{aligned}
$$


Similarly for $l=0, \ldots, i$

$$
E_{t_{l}}\left(r_{j_{l}}, \ldots, r_{j_{l}}\right)=\sum_{k=0}^{t_{l}-1} E\left(r_{j_{l}}\right) q\left(r_{j_{l}}\right)^{k}=T\left(r_{j_{l}}, t_{l}\right)
$$

which completes the proof.

\section{APPENDIX V}

\section{Proof of Lemma 4}

Using Lemma 3, we have for $i \geq 0$

$$
\begin{aligned}
& \left(A_{i}-B_{i}\right) q\left(r_{j_{i}}\right)^{t-1} \\
& =\frac{E_{1}\left(r_{j_{i}}\right)}{1-q\left(r_{j_{i}}\right)}-T\left(r_{j_{i}}, t-1\right)-q\left(r_{j_{i}}\right)^{t-1} \\
& \quad \times \sum_{l=0}^{i-1} T\left(r_{j_{l}}, t_{l}\right) \prod_{k=l+1}^{i-1} q\left(r_{j_{k}}\right)^{t_{k}} \\
& =\frac{E_{1}\left(r_{j_{i}}\right)}{1-q\left(r_{j_{i}}\right)}-E(\underbrace{r_{j_{i}}, \ldots, r_{j_{i}}}_{t-1}, \underbrace{r_{j_{i-1}}^{i_{j}}, \ldots, r_{j_{i-1}}}_{t_{i-1}}, \\
& \quad \ldots, \underbrace{r_{j_{0}}, \ldots, r_{j_{0}}}_{t_{0}})
\end{aligned}
$$

where for $i=0$, the expression

$$
E(\underbrace{r_{j_{i}}, \ldots, r_{j_{i}}}_{t-1}, \underbrace{r_{j_{i-1}}, \ldots, r_{j_{i-1}}}_{t_{i-1}}, \ldots, \underbrace{r_{j_{0}}, \ldots, r_{j_{0}}}_{t_{0}})
$$

reads $E(\underbrace{r_{j_{0}}, \ldots, r_{j_{0}}}_{t-1})$. Thus, the sign of $\psi_{i, k}(t)$ is equal to that of

$$
\begin{aligned}
E_{1}\left(r_{j_{i}}\right)-E_{1}\left(r_{k}\right)-\left(p\left(r_{j_{i}}\right)\right. & \left.-p\left(r_{k}\right)\right) \\
& \times E(\underbrace{r_{j_{i}}, \ldots, r_{j_{i}}}_{t-1}, \ldots, \underbrace{r_{j_{0}}, \ldots, r_{j_{0}}}_{t_{0}})
\end{aligned}
$$

or, equivalently, to that of

$$
\begin{aligned}
{\left[E_{1}\left(r_{j_{i}}\right)-\right.} & \left.p\left(r_{j_{i}}\right) E(\underbrace{r_{j_{i}}, \ldots, r_{j_{i}}}_{t-1}, \ldots, \underbrace{r_{j_{0}}, \ldots, r_{j_{0}}}_{t_{0}})\right] \\
& -\left[E_{1}\left(r_{k}\right)-p\left(r_{k}\right) E(\underbrace{r_{j_{i}}, \ldots, r_{j_{i}}}_{t-1}, \ldots, \underbrace{r_{j_{0}}, \ldots, r_{j_{0}}}_{t_{0}})\right]
\end{aligned}
$$

which, by adding $E(\underbrace{r_{j_{i}}, \ldots, r_{j_{i}}}_{t-1}, \ldots, \underbrace{r_{j_{0}}, \ldots, r_{j_{0}}}_{t_{0}})$ to the two terms between the brackets, is the sign of

$$
\begin{aligned}
& E(r_{j_{i}}, \underbrace{r_{j_{i}}, \ldots, r_{j_{i}}}_{t-1}, \ldots, \underbrace{r_{j_{0}, \ldots, r_{j_{0}}}, \ldots}_{t_{0}}) \\
&-E(r_{k}, \underbrace{r_{j_{i}}, \ldots, r_{j_{i}}}_{t-1}, \ldots, \underbrace{r_{j_{0}}, \ldots, r_{j_{0}}}_{t_{0}})
\end{aligned}
$$

APPENDIX VI

Proof of Lemma 5

$$
\begin{aligned}
A_{i}-B_{i}= & \frac{E_{1}\left(r_{j_{i}}\right)}{1-q\left(r_{j_{i}}\right)} \\
& -E(\underbrace{r_{j_{i-1}}, \ldots, r_{j_{i-1}}}_{t_{i-1}}, \ldots, \underbrace{r_{j_{0}}, \ldots, r_{j_{0}}}_{t_{0}}) \\
= & \frac{1}{1-q\left(r_{j_{i}}\right)} \\
& \times\left(E_{1}\left(r_{j_{i}}\right)+q\left(r_{j_{i}}\right)\right. \\
& \left.\times E(\underbrace{r_{j_{i-1}}, \ldots, r_{j_{i-1}}}_{t_{i-1}}, \ldots, \underbrace{r_{j_{0}}, \ldots, r_{j_{0}}}_{t_{0}})\right) \\
& -\frac{1}{1-q\left(r_{j_{i}}\right)} E(\underbrace{r_{j_{i-1}}, \ldots, r_{j_{i-1}}}_{t_{i-1}}, \ldots, \underbrace{r_{j_{0}}, \ldots, r_{j_{0}}}_{t_{0}}) \\
= & \frac{1}{1-q\left(r_{j_{i}}\right)} \quad(r_{j_{i}}, \underbrace{r_{j_{i-1}}, \ldots, r_{j_{i-1}}}_{t_{i-1}}, \ldots, \underbrace{r_{j_{0}}, \ldots, r_{j_{0}}}_{t_{0}}) \\
& \left.\quad-E(\underbrace{r_{j_{i-1}}, \ldots, r_{j_{i-1}}}_{t_{i-1}}, \ldots, \underbrace{r_{j_{0}, \ldots, r_{j_{0}}}}_{t_{0}})\right) .
\end{aligned}
$$

Since $(\underbrace{r_{j_{i-1}}, \ldots, r_{j_{i-1}}}_{t_{i-1}}, \ldots, \underbrace{r_{j_{0}}, \ldots, r_{j_{0}}}_{t_{0}})$ is rate optimal, the $\operatorname{EPS}(r_{j_{i-1}}, \underbrace{r_{j_{i-1}}, \ldots, r_{j_{i-1}}}_{t_{i-1}}, \ldots, \underbrace{r_{j_{0}}, \ldots, r_{j_{0}}}_{t_{0}})$ is rate optimal over all EPSs whose first code rate is constrained to be $r_{j_{i-1}}$. Thus, by Proposition 1.2, (11) gives

$$
\begin{aligned}
A_{i}-B_{i} \geq & \frac{1}{1-q\left(r_{j_{i}}\right)} \\
& \times\left(E(r_{j_{i}}, \underbrace{r_{j_{i-1}}, \ldots, r_{j_{i-1}}}_{t_{i-1}}, \ldots, \underbrace{r_{j_{0}}, \ldots, r_{j_{0}}}_{t_{0}})\right. \\
& \left.-E(r_{j_{i-1}}, \underbrace{r_{j_{i-1}}, \ldots, r_{j_{i-1}}}_{t_{i-1}}, \ldots, \underbrace{r_{j_{0}}, \ldots, r_{j_{0}}}_{t_{0}})\right) \\
> & 0
\end{aligned}
$$

where the last inequality is obtained from Lemma 4 using the assumption $\psi_{i-1, j_{i}}\left(t_{i-1}+1\right)<0$.

\section{ACKNOWLEDGMENT}

The authors thank M. Röder for providing his RCPC coder. They also thank M. Zhao and B. Banister for fruitful discussions.

\section{REFERENCES}

[1] P. G. Sherwood and K. Zeger, "Progressive image coding for noisy channels," IEEE Signal Processing Lett., vol. 4, pp. 191-198, July 1997.

[2] V. Chande and N. Farvardin, "Progressive transmission of images over memoryless noisy channels," IEEE J. Select. Areas Commun., vol. 18 , pp. $850-860$, June 2000

[3] A. Said and W. A. Pearlman, "A new fast and efficient image codec based on set partitioning in hierarchical trees," IEEE Trans. Circuits Syst. Video Technol., vol. 6, pp. 243-250, June 1996. 
[4] D. Taubman and M. Marcellin, JPEG2000: Image Compression Fundamentals, Standards, and Practice. Norwell, MA: Kluwer, 2001.

[5] B.-J. Kim, Z. Xiong, and W. A. Pearlman, "Low bit-rate scalable video coding with 3D set partitioning in hierarchical trees (3D SPIHT)," IEEE Trans. Circuits Syst. Video Technol., vol. 10, pp. 1365-1374, Dec. 2000.

[6] M: Zhao, A. A. Alatan, and A. N. Akansu, "A new method for optimal rate allocation for progressive image transmissions over noisy channels," in Proc. Data Compression Conf., Snowbird, UT, Mar. 2000, pp. 213-222.

[7] A. Hedayat and A. Nosratinia, "Rate allocation in source-channel coding of images," in Proc. IEEE Int. Conf. Image Processing, vol. I, Thessaloniki, Greece, Oct. 2001, pp. 189-192.

[8] R. Hamzaoui, V. Stanković, and Z. Xiong, "Rate-based versus distortionbased optimal joint source-channel coding," in Proc. Data Compression Conf., Snowbird, UT, Apr. 2002, pp. 63-72.

[9] B. A. Banister, B. Belzer, and T. R. Fischer, "Robust image transmission using JPEG2000 and turbo codes," IEEE Signal Processing Lett., vol. 9, pp. 117-119, Apr. 2002.

[10] D. N. Rowitch and L. B. Milstein, "On the performance of hybrid FEC/ARO systems using rate compatible punctured turbo (RCPT) codes," IEEE Trans. Commun., vol. 48, pp. 948-959, June 2000.

[11] B. Vucetic and J. Yuan, Turbo Codes: Principles and Applications. Norwell, MA: Kluwer, 2000.

[12] V. Stanković, R. Hamzaoui, and D. Saupe, "Fast algorithm for optima error protection of embedded wavelet codes," in Proc. IEEE Workshop Multimedia Signal Processing, Cannes, France, Oct. 2001, pp. 593-598.

[13] S. Lin and D. J. Costello, Jr., Error Control Coding. Englewood Cliffs, NJ: Prentice-Hall, 1983

[14] F. K. Song and E. F. Huang "A tree-trellis-based fast search algorithm for finding the $N$ best sentence hypotheses in continuous speech recognition," in Proc. IEEE Int. Conf. Acoustics, Speech, Signal Processing, Toronto, ON, Canada, May 1991, pp. 705-708.

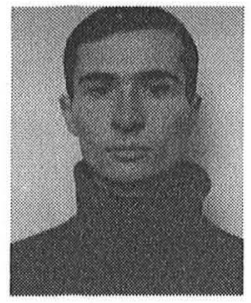

Vladimir Stanković received the Dipl.-Ing. degree in electrical engineering from the University of Belgrade, Belgrade, Serbia, in 2000, and the Dr.-Ing. degree from the University of Leipzig, Leipzig, Germany, in 2003.

From 2002 to 2003, he was with the Department of Computer and Information Science, University of Konstanz, Konstanz, Germany. He is currently a Postdoctoral Research Associate with the Department of Electrical Engineering, Texas A\&M University, College Station. His research interests include robust image and video transmission, error protection coding, and data compression.

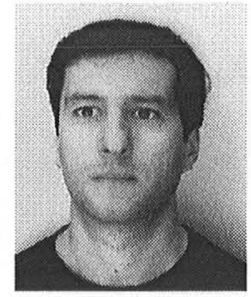

Raouf Hamzaoui received the Maîtrise de mathématiques from the Faculty of Sciences of Tunis, Tunis, Tunisia, in 1986, the M.Sc. degree in mathematics from the University of Montreal, Montreal, QC, Canada, in 1993, and the Dr. rer. nat. degree from the Faculty of Applied Sciences, University of Freiburg, Freiburg, Germany, in 1997.

From 1998 to 2002, he was a Research Assistant with the Computer Science Department, University of Leipzig, Leipzig, Germany. He is currently a Research Assistant with the Department of Computer and Information Science, University of Konstanz, Konstanz, Germany. His research interests include data compression and joint source-channel coding.

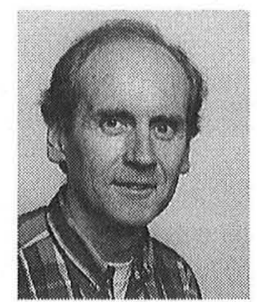

Dietmar Saupe received the Dr. rer. nat. and Habilitation degrees, both from the University of Bremen, Bremen, Germany, in 1982 and 1993, respectively.

$\mathrm{He}$ served as Visiting Assistant Professor of Mathematics at the University of California at Santa Cruz (1985-1987), Assistant Professor at the University of Bremen (1987-1993), Professor of Computer Science at the Albert-Ludwigs-University of Freiburg (1993-1998), at the University of Leipzig (1998-2002), and at the University of Konstanz (since 2002). His research has focused on image processing and coding, computer graphics, visualization, and dynamical systems. He is the co-author and editor of several books on fractals, e.g., Chaos and Fractals (Springer-Verlag, New York, 1992).

Dr Saupe is a member of the IEEE Signal Processing Society, ACM SIGGRAPH, Eurographics, and others. 